\title{
Percutaneous Coronary Intervention for High Takeoff Left Main Coronary Artery: A Case Report
}

\author{
Seetharam Vankudoth ${ }^{1}$ Madhurima Banoth² \\ ${ }^{1}$ Department of Cardiology, Nizam's Institute of Medical Sciences, \\ Hyderabad, Telangana, India \\ 2Osmania Medical College, Hyderabad, Telangana, India
}

\begin{abstract}
Address for correspondence Seetharam Vankudoth, DM Student, Department of Cardiology, Nizam's Institute of Medical Sciences, Hyderabad 500082, Telangana, India (e-mail: sitaramdm14@gmail.com).
\end{abstract}

Indian J Cardiovasc Dis Women-WINCARS 2018;3:237-239

\begin{abstract}
Keywords

- high takeoff

- left main

- percutaneous coronary intervention

- post-myocardial infarction ventricular septal defect

Percutaneous coronary intervention ( $\mathrm{PCl}$ ) for high takeoff left main is challenging, as it poses difficulties with the engagement of the guiding catheter and establishment of backup support. This report examines the case of a 53-year-old woman with history of anterior wall myocardial infarction with a ventricular septal defect (VSD), who was treated with left anterior descending (LAD) angioplasty and VSD device closure done 4 years back, and now she presented with unstable angina. After successful engagement of $5 \mathrm{~F}$ Tiger diagnostic catheter through a right radial artery, the angiography revealed an $80 \%$ stenosis of the proximal LAD and in-stent restenosis $70 \%$ of mid$L A D$. The authors tried to engage the left coronary system through the right femoral artery with $6 \mathrm{~F}$ judkins left, $6 \mathrm{~F}$ Amplatzer left, $6 \mathrm{~F} \mathrm{EBU,} \mathrm{and} \mathrm{6F} \mathrm{XBU.} \mathrm{They} \mathrm{could} \mathrm{not}$ cannulate because of high takeoff left main, so they switched to right radial access. Then they engaged a 6F 3.5 EBU catheter. Due to the weak backup support of the guiding catheter, they used another wire to stabilize it and the stent was implanted successfully. This is one of the rare case reports of PCI for high takeoff left main.
\end{abstract}

\section{Introduction}

Commonly, the coronary arteries arise from the aortic sinuses rather than from the tubular aorta. Often, however, the coronary arteries are located above the tubular junction, usually by only a few millimeters. ${ }^{1}$ Very high takeoff of the coronary artery, well above the sinotubular junction, occurs in less than $1 \%$ of the population, ${ }^{2}$ and data concerning this rare finding are confined to scattered case reports. Due to the difficulty in correctly engaging the guiding catheter and in gaining backup support, the performance of PCI for a high takeoff left main is challenging. We report a case of unstable angina of high takeoff the left main in which the patient underwent a successful PCI.

\section{Case Report}

A 53-year-old woman presented to our outpatient department with a complaint of chest pain and dyspnea for 1 week. She had a history of anterior wall myocardial infarction (MI) with a post-MI ventricular septal defect (VSD). She underwent left anterior descending (LAD) angioplasty and VSD device closure. Her physical examination was remarkable. On electrocardiography, there was ST-depression in anterior leads. On echocardiography, there was hypokinesia in LAD territory, moderate left ventricular (LV) dysfunction, and VSD device in situ with no residual shunt. The patient was admitted and scheduled for coronary angiography. Several attempts to cannulate the coronary arteries were unsuccessful ( - Fig. 1). We tried to engage the left coronary system through the right femoral artery with $6 \mathrm{~F}$ Judkins left, $6 \mathrm{~F}$ Amplatzer, 6F EBU, and 6 F XBU. We could not engage because of high takeoff left main, so we switched to right radial access. Then we could engage a 6F 3.5 EBU catheter ( - Fig. 2). Due to the weak backup support of the guiding catheter, we used another wire to stabilize it, and the stent was implanted successfully.

This is one of the rare case reports of percutaneous coronary intervention $(\mathrm{PCI})$ for high takeoff left main. The patient improved symptomatically, was hemodynamically stable, and was discharged after 4 days. 

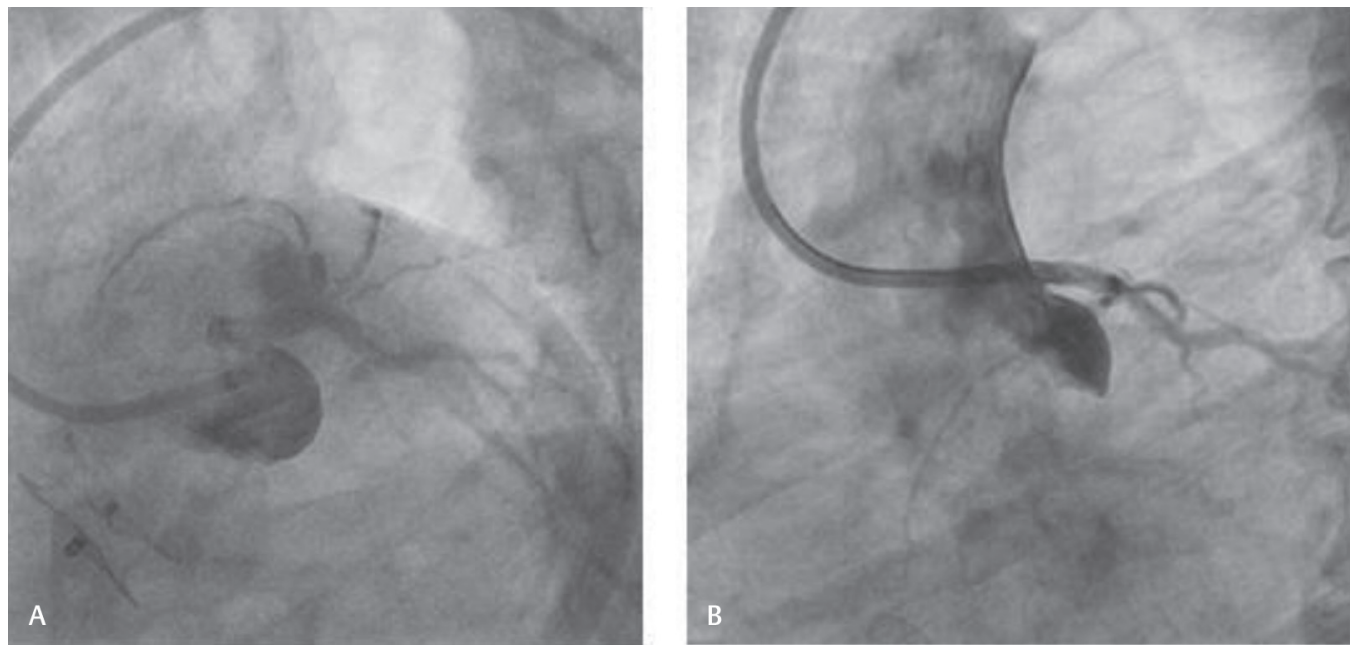

Fig. 1 (A and B) High takeoff left main through the right femoral artery.
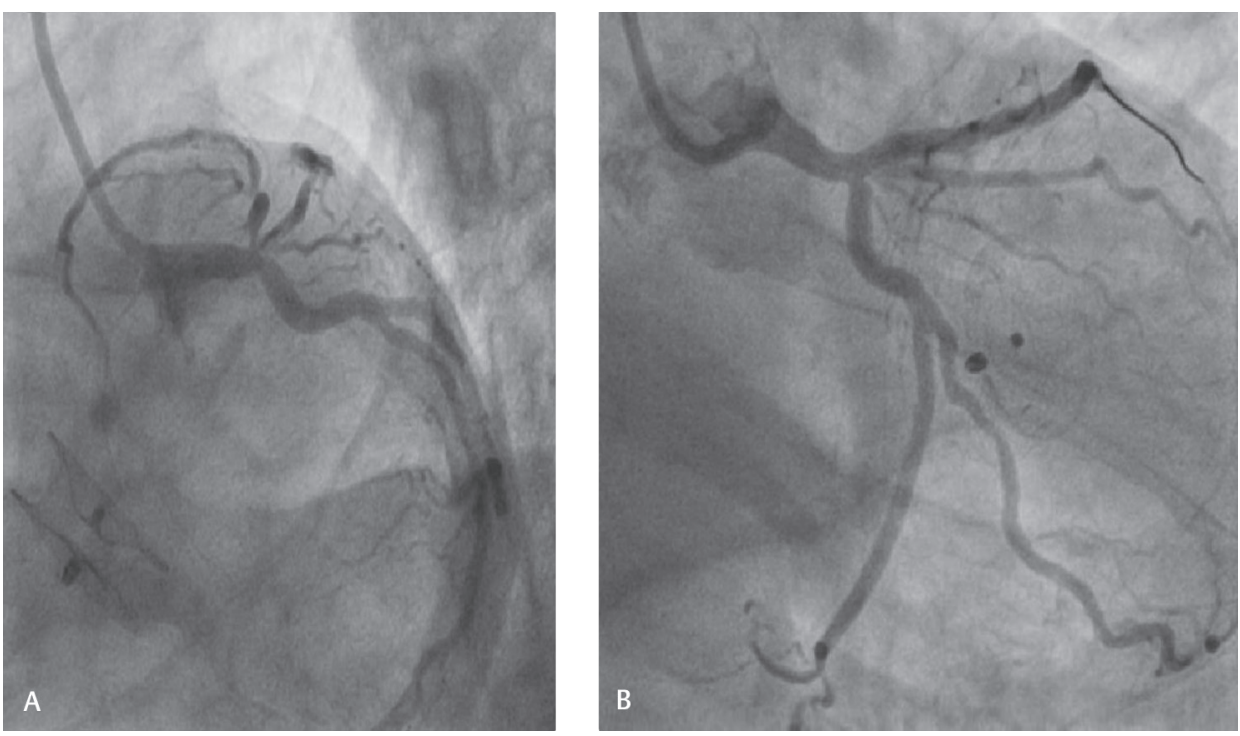

Fig. 2 (A and B) Pre- and post-angioplasty through the right radial artery.

\section{Discussion}

The overall incidence of anomalous origin of the left main coronary artery (LMCA) above the coronary sinus has ranged from 0.019 to $0.04 \%$ in catheterized populations. ${ }^{3,4}$ In many cases, this seems to be a normal variant. In $44 \%$ of the cases examined by Vlodaver and colleagues, ${ }^{5}$ one or both of the coronary ostia rose above the sinotubular junction: most frequently the LMCA (30\%), followed by the right coronary artery ( $8 \%$ ) and by both vessels (6\%). A "high takeoff" is variably defined in the literature. Some authors classify any coronary artery with its origin above the sinotubular junction as anomalous. However, this definition appears to be inappropriately broad, because it includes many anatomically normal variants. Other authors restrict the definition of high takeoff to ostia that are more than 1 or $2 \mathrm{~cm}$ above the sinotubular junction. The highest reported origin of a coronary artery has been $5 \mathrm{~cm}$ above the sinotubular junction, in a case involving a right coronary artery. ${ }^{6}$
Although reports of PCI for high takeoff left main are scarce, in our case, the performance of PCI for high takeoff left main was challenging due to the difficulty in correctly engaging the guiding catheter and in gaining backup support. We were unable to engage an Amplatz-Left 0.75 guiding catheter. Although an Ikari-Left (Terumo) catheter would have been a better option to consider, as it is sometimes suitable for PCIs for right coronary lesions, including in cases of high takeoff ostium, ${ }^{7}$ we eventually performed the $\mathrm{PCI}$ with a $6 \mathrm{~F}$ EBU from right radial access. However, because of the non-coaxial guiding catheter, the backup support for delivery of the stent was insufficient. We inserted a run through wire in the left circumflex artery (LCX) for enhanced support. In this type of difficult case, a change in access route is an option for engagement of the catheter. ${ }^{8}$

Previously, coronary angiography was the gold standard for the diagnosis of coronary artery anomalies. However, recent reports found that correct identification of these anomalies is feasible in only $53 \%$ of cases compared with 
computed tomographic (CT) angiography study. ${ }^{9}$ According to the modified classification of Greenberg et al, ${ }^{10}$ coronary artery anomalies are classified into three main groups: anomalous origin, anomalous course, and anomalous termination. Anomalous origin group includes high takeoff arteries, multiple ostia, origin from the pulmonary artery, and origin from opposite sinus with the abnormal course. In our case, the LMCA and right coronary artery originated from the left aortic side, and above sinotubular junction correlate with high takeoff definition.

High takeoff coronary arteries can present challenges during catheter cannulation. During coronary bypass surgery, if the anomaly has not been recognized, there have been cases of inadvertent transection and coronary cross-clamping during open-heart procedures. ${ }^{11} \mathrm{CT}$ coronary angiography enables a precise display of the relationship between the coronary ostia and aortic sinuses and more easily defines anomalous origins of vessels than does conventional invasive angiography. ${ }^{12}$ When a high takeoff coronary artery ostia is identified, CT coronary angiography appears to be ideally suited to determine the ostium and course of the vessel further, thereby enabling the discovery (or exclusion) of abnormalities that might produce ischemia.

\section{Conflict of Interest}

None declared.

\section{References}

1. Muriago M, Sheppard MN, Ho SY, Anderson RH. Location of the coronary arterial orifices in the normal heart. Clin Anat 1997;10(5):297-302

2. Angelini P, Velasco JA, Flamm S. Coronary anomalies: incidence, pathophysiology, and clinical relevance. Circulation 2002;105(20):2449-2454
3. Yamanaka O, Hobbs RE. Coronary artery anomalies in 126,595 patients undergoing coronary arteriography. Cathet Cardiovasc Diagn 1990;21(1):28-40

4. Mavi A, Ayalp R, Serçelik A, Pesțemalci T, Batyraliev T, Gümüşburun E. Frequency in the anomalous origin of the left main coronary artery with angiography in a Turkish population. Acta Med Okayama 2004;58(1):17-22

5. Vlodaver Z, Neufeld HN, Edwards JE. Pathology of coronary disease. Semin Roentgenol 1972;7(4):376-394

6. Motamedi MH, Hemmat A, Kalani P, Rezaee MR, Safarnezhad S. High take-off of right coronary artery: an extremely rare case of RCA anomaly. J Card Surg 2009;24(3):343-345

7. Youssef AA, Hsieh YK, Cheng $\mathrm{CI}, \mathrm{Wu} \mathrm{CJ}$. A single transradial guiding catheter for right and left coronary angiography and intervention. Eurolntervention 2008;3(4):475-481

8. Kawashima O, Endoh N, Terashima M, et al. Effectiveness of right or left radial approach for coronary angiography. Randomized controlled trial. Catheter Cardiovasc Interv 2004;61(3):333-337

9. Shi H, Aschoff AJ, Brambs HJ, Hoffmann MH. Multislice CT imaging of anomalous coronary arteries. Eur Radiol 2004; 14(12):2172-2181

10. Greenberg MA, Fish BG, Spindola-Franco H. Congenital anomalies of the coronary arteries. Classification and significance. Radiol Clin North Am 1989;27(6):1127-1146

11. Tarhan A, Kehlibar T, Yilmaz M, et al. Right coronary artery with high takeoff. Ann Thorac Surg 2007;83(5):1867-1869

12. Berbarie RF, Dockery WD, Johnson KB, Rosenthal RL, Stoler $\mathrm{RC}$, Schussler JM. Use of multislice computed tomographic coronary angiography for the diagnosis of anomalous coronary arteries. Am J Cardiol 2006;98(3):402-406 